\title{
Luminosity determination at HERA-B
}

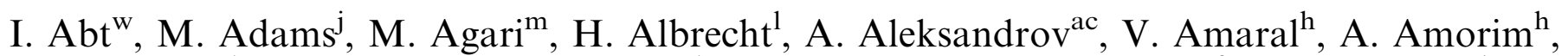
S.J. Aplin ${ }^{1}$, V. Aushev ${ }^{\mathrm{p}}$, Y. Bagaturia ${ }^{1,20}$, V. Balagura ${ }^{\mathrm{v}}$, M. Bargiotti ${ }^{\mathrm{f}}$, O. Barsukova ${ }^{\mathrm{k}}$, J. Bastos ${ }^{\mathrm{h}}$, J. Batista ${ }^{\mathrm{h}}$, C. Bauer ${ }^{\mathrm{m}}$, Th.S. Bauer ${ }^{\mathrm{a}}$, A. Belkov, ${ }^{\mathrm{k}}$, Ar. Belkov ${ }^{\mathrm{k}}$, I. Belotelov ${ }^{\mathrm{k}}$,

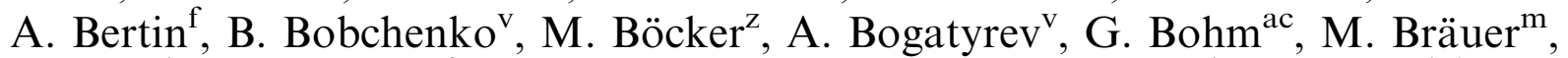

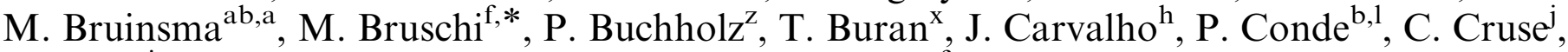
M. Dam ${ }^{\mathrm{i}}$, K.M. Danielsen ${ }^{\mathrm{x}}$, M. Danilov ${ }^{\mathrm{v}}$, S. De Castro ${ }^{\mathrm{f}}$, H. Deppe ${ }^{\mathrm{n}}$, X. Dong $^{\mathrm{c}}$, H.B. Dreis ${ }^{\mathrm{n}}$, V. Egorytchev ${ }^{1}$, K. Ehret ${ }^{\mathrm{j}}$, F. Eisele ${ }^{\mathrm{n}}$, D. Emeliyanov' ${ }^{1}$, S. Essenov ${ }^{\mathrm{v}}$, L. Fabbrif ${ }^{\mathrm{f}}$, P. Faccioli ${ }^{\mathrm{f}}$, M. Feuerstack-Raible ${ }^{\mathrm{n}}$, J. Flammer ${ }^{1}$, B. Fominykh ${ }^{\mathrm{v}}$, M. Funcke ${ }^{\mathrm{j}}$, Ll. Garrido ${ }^{\mathrm{b}}$, A. Gellrich ${ }^{\mathrm{ac}}$, B. Giacobbe ${ }^{\mathrm{f}}$, J. Gläß ${ }^{\mathrm{t}}$, D. Goloubkov ${ }^{1,17}$, Y. Golubkov ${ }^{1,18}$, A. Golutvin ${ }^{\mathrm{v}}$, I. Golutvin ${ }^{\mathrm{k}}$, I. Gorbounov ${ }^{1, z}$, A. Gorišek ${ }^{\mathrm{q}}$, O. Gouchtchine ${ }^{\mathrm{v}}$, D.C. Goulart ${ }^{\mathrm{g}}$, S. Gradl ${ }^{\mathrm{n}}$, W. Gradl ${ }^{\mathrm{n}}$, F. Grimaldif , J. Groth-Jensen ${ }^{\mathrm{i}}$, Yu. Guilitsky ${ }^{\mathrm{v}, 19}$, J.D. Hansen ${ }^{\mathrm{i}}$, J.M. Hernández ${ }^{\mathrm{ac}}$, W. Hofmann ${ }^{\mathrm{m}}$, M. Hohlmann ${ }^{1}$, T. Hott ${ }^{\mathrm{n}}$, W. Hulsbergen ${ }^{\mathrm{a}}$, U. Husemann ${ }^{\mathrm{z}}$, O. Igonkina ${ }^{\mathrm{v}}$, M. Ispiryan ${ }^{\mathrm{o}}$, T. Jagla ${ }^{\mathrm{m}}$, C. Jiang ${ }^{\mathrm{c}}$, H. Kapitzal, S. Karabekyan ${ }^{\mathrm{y}}$, N. Karpenko ${ }^{\mathrm{k}}$, S. Keller ${ }^{\mathrm{z}}$, J. Kessler ${ }^{\mathrm{n}}$, F. Khasanov ${ }^{\mathrm{v}}$, Yu. Kiryushin ${ }^{\mathrm{k}}$, I. Kisel ${ }^{\mathrm{w}}$, E. Klinkby ${ }^{\mathrm{i}}$, K.T. Knöpfle ${ }^{\mathrm{m}}$, H. Kolanoski ${ }^{\mathrm{e}}$, S. Korpar ${ }^{\mathrm{u}, \mathrm{q}}$, C. Krauss ${ }^{\mathrm{n}}$, P. Kreuzer, ${ }^{1, \mathrm{~s}}$, P. Križan ${ }^{\mathrm{r}, \mathrm{q}}$, D. Krücker ${ }^{\mathrm{e}}$, S. Kupper ${ }^{\mathrm{q}}$, T. Kvaratskheliia ${ }^{v}$, A. Lanyov ${ }^{\mathrm{k}}$, K. Lau ${ }^{\mathrm{o}}$, B. Lewendel ${ }^{1}$, T. Lohse ${ }^{\mathrm{e}}$, B. Lomonosov ${ }^{1,16}$,

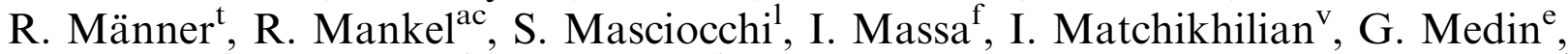
M. Medinnis, ${ }^{1}$ M. Mevius ${ }^{1}$, A. Michetti ${ }^{1}$, Yu. Mikhailov $^{\mathrm{v}, 19}$, R. Mizuk ${ }^{\mathrm{v}}$, R. Muresan, M. zur Nedden ${ }^{\mathrm{e}}$, M. Negodaev ${ }^{1,16}$, M. Nörenberg ${ }^{1}$, S. Nowak ${ }^{\text {ac }}$, M.T. Núñez Pardo de Vera ${ }^{1}$, M. Ouchrif ${ }^{\mathrm{ab}, \mathrm{a}}$, F. Ould-Saada ${ }^{\mathrm{x}}$, C. Padilla ${ }^{1}$, D. Peralta ${ }^{\mathrm{b}}$, R. Pernack ${ }^{\mathrm{y}}$, R. Pestotnik ${ }^{\mathrm{q}}$, B.AA. Petersen ${ }^{\mathrm{i}}$, M. Piccininif ${ }^{\mathrm{f}}$, M.A. Pleier ${ }^{\mathrm{m}}$, M. Poli ${ }^{\mathrm{f}, 15}$, V. Popov ${ }^{\mathrm{v}}$, D. Pose ${ }^{\mathrm{k}, \mathrm{n}}$, S. Prystupa $^{\mathrm{p}}$, V. Pugatch ${ }^{\mathrm{p}}$, Y. Pylypchenko ${ }^{\mathrm{x}}$, J. Pyrlik ${ }^{\mathrm{o}}$, K. Reeves ${ }^{\mathrm{m}}$, D. Reßing ${ }^{1}$, H. Rick ${ }^{\mathrm{n}}$, I. Riul, P. Robmann ${ }^{\text {ad }}$, I. Rostovtseva ${ }^{\mathrm{v}}$, V. Rybnikov', F. Sánchez ${ }^{\mathrm{m}}$, A. Sbrizzi ${ }^{\mathrm{a}}$, M. Schmelling ${ }^{\mathrm{m}}$, B. Schmidt ${ }^{1}$, A. Schreiner ${ }^{\text {ac }}$, H. Schröder ${ }^{y}$, U. Schwanke ${ }^{\text {ac }}$, A.J. Schwartz ${ }^{g}$, A.S. Schwarz ${ }^{1}$, B. Schwenninger ${ }^{\mathrm{j}}$, B. Schwingenheuer ${ }^{\mathrm{m}}$, F. Sciacca ${ }^{\mathrm{m}}$, N. Semprini-Cesari ${ }^{\mathrm{f}}$, S. Shuvalov, ${ }^{\mathrm{v}, \mathrm{e}}$, L. Silva ${ }^{h}$, L. Sözüer ${ }^{1}$, S. Solunin ${ }^{k}$, S. Somov ${ }^{1}$, A. Somov ${ }^{1,17}$, J. Spengler ${ }^{1}$, R. Spighif ${ }^{\mathrm{f}}$,

A. Spiridonov ${ }^{\text {ac,v }}$, A. Stanovnik ${ }^{\mathrm{r}, \mathrm{q}}$, M. Starič ${ }^{\mathrm{q}}$, C. Stegmann ${ }^{\mathrm{e}}$, H.S. Subramania ${ }^{\mathrm{o}}$, M. Symalla,

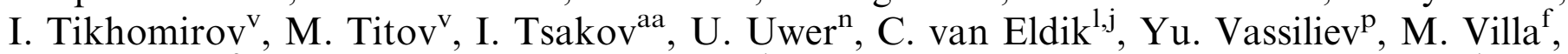

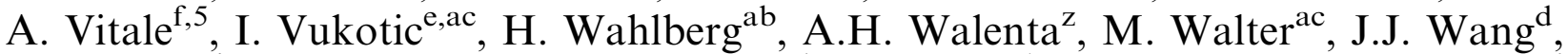
D. Wegener ${ }^{\mathrm{j}}$, U. Werthenbach ${ }^{\mathrm{z}}$, H. Wolters ${ }^{\mathrm{h}}$, R. Wurth ${ }^{1}$, A. Wurz, S. Xella-Hansen ${ }^{\mathrm{i}}$, Yu. Zaitsev ${ }^{\mathrm{v}}$, M. Zavertyaev ${ }^{1, \mathrm{~m}, 16}$, T. Zeuner, ${ }^{1, \mathrm{z}}$, A. Zhelezov ${ }^{\mathrm{v}}$, Z. Zheng ${ }^{\mathrm{c}}$, R. Zimmermann ${ }^{\mathrm{y}}$, T. Živko ${ }^{\mathrm{q}}$, A. Zoccoli ${ }^{\mathrm{f}}$

\footnotetext{
*Corresponding author.

E-mail address: Marco.Bruschi@bo.infn.it (M. Bruschi).
} 
${ }^{\mathrm{c}}$ Institute for High Energy Physics, Beijing 100039, PR China

${ }^{\mathrm{d}}$ Institute of Engineering Physics, Tsinghua University, Beijing 100084, PR China.

${ }^{\mathrm{e}}$ Institut für Physik, Humboldt-Universität zu Berlin, D-12489 Berlin, Germany ${ }^{3,}{ }^{4}$

${ }^{\mathrm{f}}$ Dipartimento di Fisica dell' Università di Bologna and INFN Sezione di Bologna, I-40126 Bologna, Italy

${ }^{\mathrm{g}}$ Department of Physics, University of Cincinnati, Cincinnati, Ohio 45221, USA ${ }^{6}$

${ }^{\mathrm{h}}$ LIP Coimbra, P-3004-516 Coimbra, Portugal ${ }^{7}$

${ }^{\mathrm{i}}$ Niels Bohr Institutet, DK 2100 Copenhagen, Denmark ${ }^{8}$

${ }_{\mathrm{j}}^{\mathrm{I} n s t i t u t}$ für Physik, Universität Dortmund, D-44221 Dortmund, Germany ${ }^{4}$

${ }^{\mathrm{k}}$ Joint Institute for Nuclear Research Dubna, 141980 Dubna, Moscow region, Russia

${ }^{1}$ DESY, D-22603 Hamburg, Germany

${ }^{\mathrm{m}}$ Max-Planck-Institut für Kernphysik, D-69117 Heidelberg, Germany ${ }^{4}$

${ }^{\mathrm{n}}$ Physikalisches Institut, Universität Heidelberg, D-69120 Heidelberg, Germany ${ }^{4}$

${ }^{\circ}$ Department of Physics, University of Houston, Houston, TX 77204, USA ${ }^{6}$

${ }^{\mathrm{p}}$ Institute for Nuclear Research, Ukrainian Academy of Science, 03680 Kiev, Ukraine ${ }^{9}$

${ }^{\mathrm{q}}$ J. Stefan Institute, 1001 Ljubljana, Slovenia ${ }^{10}$

${ }^{\mathrm{r}}$ University of Ljubljana, 1001 Ljubljana, Slovenia

${ }^{\mathrm{s}}$ University of California, Los Angeles, CA 90024, USA ${ }^{11}$

${ }^{\mathrm{t}}$ Lehrstuhl für Informatik V, Universität Mannheim, D-68131 Mannheim, Germany

${ }^{\mathrm{u}}$ University of Maribor, 2000 Maribor, Slovenia

${ }^{\mathrm{v}}$ Institute of Theoretical and Experimental Physics, 117218 Moscow, Russia ${ }^{12}$

${ }^{\mathrm{w}}$ Max-Planck-Institut für Physik, Werner-Heisenberg-Institut, D-80805 München, Germany ${ }^{4}$

${ }^{\mathrm{x}}$ Department of Physics, University of Oslo, N-0316 Oslo, Norway ${ }^{13}$

${ }^{\mathrm{y}}$ Fachbereich Physik, Universität Rostock, D-18051 Rostock, Germany ${ }^{4}$

${ }^{\mathrm{z}}$ Fachbereich Physik, Universität Siegen, D-57068 Siegen, Germany ${ }^{4}$

${ }^{\text {aa } I n s t i t u t e}$ for Nuclear Research, INRNE-BAS, Sofia, Bulgaria

${ }^{\mathrm{ab}}$ Universiteit Utrecht/NIKHEF, $3584 C B$ Utrecht, The Netherlands ${ }^{1}$

${ }^{\mathrm{ac}}$ DESY, D-15738 Zeuthen, Germany

${ }^{\text {ad }}$ Physik-Institut, Universität Zürich, CH-8057 Zürich, Switzerland ${ }^{14}$

Received 1 June 2007; received in revised form 3 September 2007; accepted 5 September 2007

Available online 12 September 2007

\footnotetext{
${ }^{1}$ Supported by the Foundation for Fundamental Research on Matter (FOM), 3502 GA Utrecht, The Netherlands.

${ }^{2}$ Supported by the CICYT Contract AEN99-0483.

${ }^{3}$ Supported by the German Research Foundation, Graduate College GRK 271/3.

${ }^{4}$ Supported by the Bundesministerium für Bildung und Forschung, FRG, under contract numbers 05-7BU35I, 05-7DO55P, 05-HB1HRA, 05-HB1KHA, 05-HB1PEA, 05-HB1PSA, 05-HB1VHA, 05-HB9HRA, 05-7HD15I, 05-7MP25I, 05-7SI75I.

${ }^{5}$ Also from Fondazione Giuseppe Occhialini, I-61034 Fossombrone(Pesaro Urbino), Italy.

${ }^{6}$ Supported by the U.S. Department of Energy (DOE).

${ }^{7}$ Supported by the Portuguese Fundação para a Ciência e Tecnologia under the program POCTI.

${ }^{8}$ Supported by the Danish Natural Science Research Council.

${ }^{9}$ Supported by the National Academy of Science and the Ministry of Education and Science of Ukraine.

${ }^{10}$ Supported by the Ministry of Education, Science and Sport of the Republic of Slovenia under contracts number P1-135 and J1-6584-0106.

${ }^{11}$ Supported by the U.S. National Science Foundation Grant PHY-9986703.

${ }^{12}$ Supported by the Russian Ministry of Education and Science, Grant SS-1722.2003.2, and the BMBF via the Max Planck Research Award.

${ }^{13}$ Supported by the Norwegian Research Council.

${ }^{14}$ Supported by the Swiss National Science Foundation.

${ }^{15}$ Visitor from Dipartimento di Energetica dell' Università di Firenze and INFN Sezione di Bologna, Italy.

${ }^{16}$ Visitor from P.N. Lebedev Physical Institute, 117924 Moscow B-333, Russia.

${ }^{17}$ Visitor from Moscow Physical Engineering Institute, 115409 Moscow, Russia.

${ }^{18}$ Visitor from Moscow State University, 119992 Moscow, Russia.

${ }^{19}$ Visitor from Institute for High Energy Physics, Protvino, Russia.

${ }^{20}$ Visitor from High Energy Physics Institute, 380086 Tbilisi, Georgia.

"Deceased.
} 


\begin{abstract}
A detailed description of an original method used to measure the luminosity accumulated by the HERA-B experiment for a data sample taken during the 2002-2003 HERA running period is reported. We show that, with this method, a total luminosity measurement can be achieved with a typical precision, including overall systematic uncertainties, at a level of $5 \%$ or better. We also report evidence for the detection of $\delta$-rays generated in the target and comment on the possible use of such delta rays to measure luminosity.

(C) 2007 Elsevier B.V. All rights reserved.
\end{abstract}

PACS: $25.60 ; 29.27 .-\mathrm{a}$

Keywords: Fixed target experiments; Luminosity determination; Delta rays; Proton nucleon collisions

\section{Introduction}

A precise determination of the luminosity is required for the measurement of absolute cross-sections. The integrated luminosity $(\mathscr{L})$ is defined by

$\mathscr{L}=\frac{N_{\mathrm{P}}}{\sigma_{\mathrm{P}}}$

where $N_{\mathrm{P}}$ is the number of events of a given process and $\sigma_{\mathrm{P}}$ is the corresponding cross-section. In the case of HERA-B, which is a forward spectrometer [1,2] experiment, operated at the $920 \mathrm{GeV}$ proton beam of the HERA accelerator at the DESY Laboratory in Hamburg, the proton beam is bunched and interacts with a nuclear target placed on the halo of the beam. The number of proton-nucleus (pA) interactions per bunch crossing is subject to statistical fluctuations. For HERA-B, as for all other experiments having a bunched beam, the luminosity can be expressed as

$\mathscr{L}=\frac{N_{B X} \cdot \lambda}{\sigma}$

where $\lambda$ is the average number of interactions per bunch crossing $B X, N_{B X}$ is the number of beam bunches crossing the apparatus and $\sigma$ is the interaction cross-section (for a more detailed discussion, see Sections 4 and 5). As a consequence, given the cross-section of proton-nucleus interactions, the luminosity can be measured by determining $\lambda$ and $N_{B X}$. The average number of interactions per $B X$ can be obtained from a fully unbiased sample of events in various ways: by looking at inclusive quantities which are proportional to the number of interactions in one event (such as the number of tracks or the energy released in a calorimeter), by counting the number of primary vertices or by counting the number of empty events. The first method has the advantage of entailing only a rather straightforward analysis of the data, but the signal corresponding to a single interaction must be evaluated precisely and detector stability becomes a relatively critical issue. In the second method, the vertex reconstruction efficiencies must be known precisely as well as the probability of erroneously merging or splitting primary vertices during reconstruction. In the third method, the distribution of the number of interactions per bunch crossing must be either known or assumed and the efficiency for detecting non-empty events and the impact of noise events must be evaluated.

After careful studies the HERA-B Collaboration has decided to exploit the method based on counting events with evidence of at least one interaction (which is equivalent to the third method listed above), since this method minimizes the systematic error on the luminosity determination allowing to achieve a final precision of about $5 \%$.

The paper is organized in the following way. In Sections 2 and 3 the main features of the HERA accelerator relevant for this analysis and the HERA-B detector are briefly described. Section 4 summarizes all of the published proton-nucleus cross-section measurements which are used for the luminosity determination. In Sections 5 and 6, the relevant relations for the determination of the luminosity are described. In Section 7 we discuss the systematic uncertainties and comment on delta ray production, while in Section 8 we report the results obtained for the interaction trigger (defined below) data sample.

\section{The HERA accelerator and the target}

HERA is a double storage ring designed for colliding a $920 \mathrm{GeV}$ proton beam with a $27.5 \mathrm{GeV}$ electron beam. Four interaction regions exist: two of them house the general purpose $e p$ detectors $\mathrm{H} 1$ and ZEUS, while the other two accommodate the fixed target experiments HERA-B and HERMES. In the following we describe the beam parameters and the filling scheme used during the HERA-B data taking period 2002-2003.

The typical proton current is $80 \mathrm{~mA}$, distributed over 180 bunches with a typical bunch length of $1-2 \mathrm{~ns}$. The proton bunches are organized into $3 \times 6$ trains of 10 consecutive bunches each, separated by one empty RF bucket. The detailed filling scheme is shown in Fig. 1. In total there are $220 \mathrm{RF}$ buckets with a spacing of $96 \mathrm{~ns}$ including a gap of 15 empty buckets at the end to provide for a secure beam dump. The average rate of filled bunch crossings is $8.52 \mathrm{MHz}$.

The target system [3] consists of two stations of four wires each. The wires are positioned above, below, and on either side of the beam and are made from various materials including carbon, titanium and tungsten. Both titanium and tungsten targets are wires with a 
diameter of $50 \mu \mathrm{m}$, whereas the carbon target is a flat ribbon, $100 \mu \mathrm{m}$ perpendicular and $500 \mu \mathrm{m}$ along the proton beam. The stations are separated by $40 \mathrm{~mm}$ along the beam direction. The wires are positioned individually in the halo of the stored proton beam and the interaction rate for each inserted wire is adjusted independently. Any number of wires can be operated simultaneously. The luminosity measurement described herein applies exclusively to single wire runs.

The steering of the target wires requires a fast and reliable system to provide a counting rate proportional to the interaction rate up to the highest interaction rates envisaged in the HERA-B design $(40 \mathrm{MHz})$. This is achieved by limiting the acceptance of the scintillation counters used to detect interactions to $\sim 10^{-2}$. Stepping motors with a nominal step-size of $50 \mathrm{~nm}$ controlled by a $10 \mathrm{~Hz}$ steering loop provide a stable interaction rate.

One additional complication is that a fraction (typically a few percent) of interactions not correlated to any bunch [4] was present. These interactions are due to so-called coasting beam protons which have left the separatrix, but are still circulating inside the machine, forming a component of the beam halo. Based on test measurements, the coasting beam can be regarded as a DC-current. The

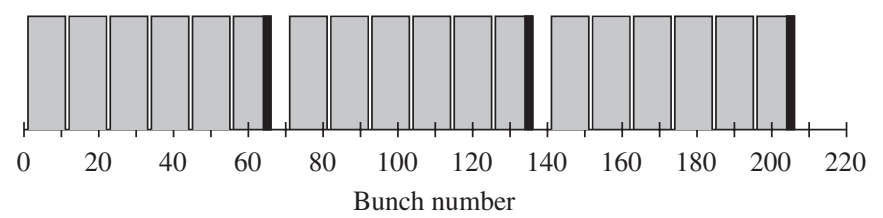

Proton fill: 180 filled buckets (dark), including 6 pilot bunches (bright) $15 \times 96$ ns gap

2x480 ns gap

$1 \times 1440$ ns gap

Fig. 1. Schematic representation of the bunch structure of a HERA proton-ring fill. fraction of coasting beam depends on the position of the target and the history of the individual proton fill, thus requiring an individual correction for each run. As described in Section 7.6, the relevant information can be derived from events triggered by a pseudo-random generator.

\section{The HERA-B detector and the data sample}

The HERA-B experiment is a forward magnetic spectrometer with an acceptance extending from 15 to $220 \mathrm{mrad}$ horizontally and to $160 \mathrm{mrad}$ vertically. This large angular coverage allows studies in kinematic regions not accessible to previous fixed-target high energy experiments. A top view of the detector is shown in Fig. 2.

The first part of the spectrometer is devoted to tracking and vertex measurements and consists of the target, a silicon vertex detector, a magnet and a tracking system. The second part is focused on particle identification and includes a Ring Imaging Cherenkov detector, an electromagnetic calorimeter and a muon detector.

The vertex detector (VDS) [5] is placed between the target and the magnet and divided in eight stations. Each station consists of four "quadrants" equipped with two double-sided silicon microstrip detectors $\left(50 \times 70 \mathrm{~mm}^{2}\right.$, $50 \mu \mathrm{m}$ pitch) each. This system provides a primary vertex resolution of $\sigma_{z} \sim 500 \mu \mathrm{m}$ along the beam direction and $\sigma_{x, y} \sim 50 \mu \mathrm{m}$ in the transverse plane.

A dipole magnet with a $2.13 \mathrm{Tm}$ field-integral is positioned before the main tracking system. Each tracking station consists of several planes of MSGC/GEM chambers placed near the beam pipe (Inner Tracker, ITR) [6] and several planes of Honeycomb Drift chambers which cover the rest of the acceptance (Outer Tracker, OTR) [7]. The detector segmentation is designed to cope with the

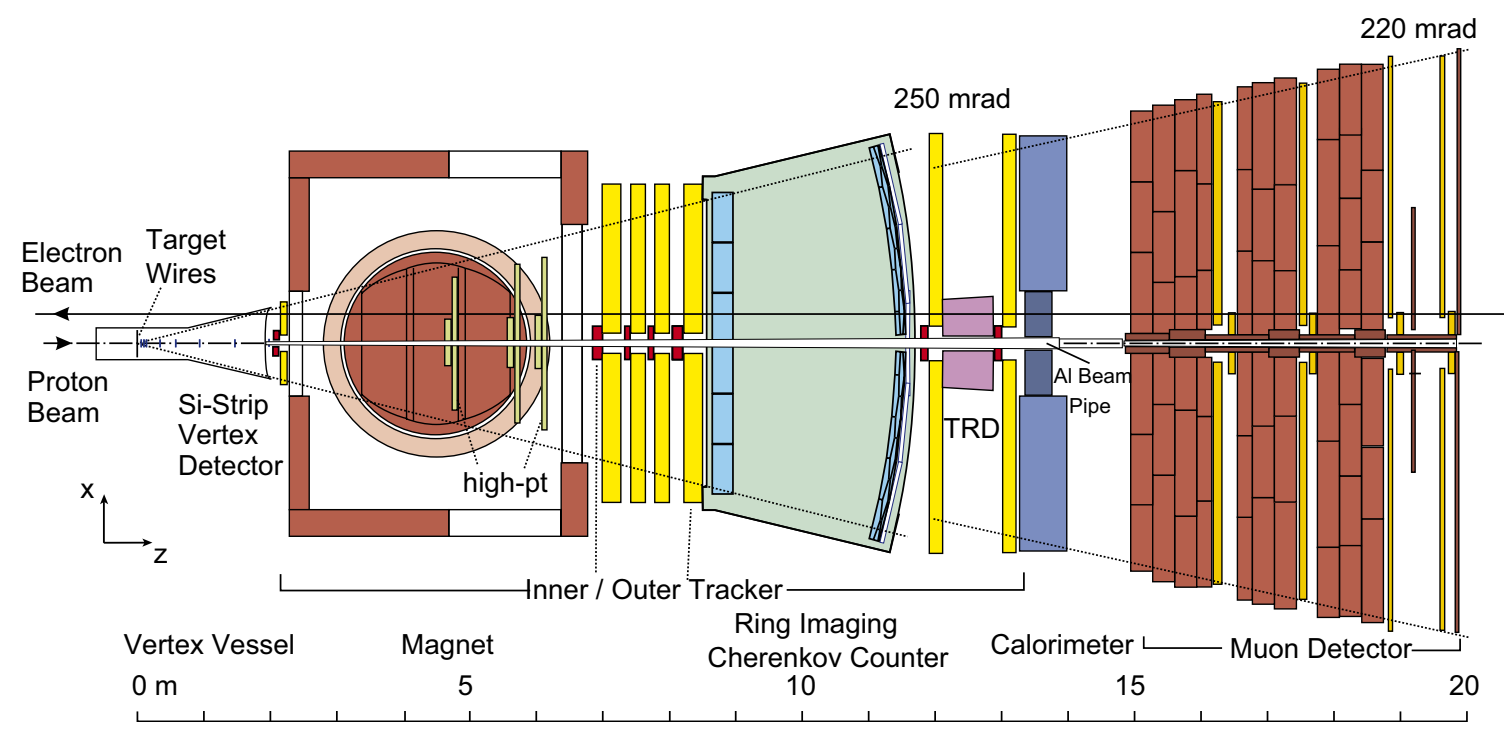

Fig. 2. Top view of the HERA-B detector. 
particle flux variation with the distance from the beam pipe. Typical momentum resolutions of $\delta_{p} / p \sim 1 \%$ are achieved.

The particle identification of charged tracks (protons, kaons, etc.) is provided by a Cherenkov detector ( $\mathrm{RICH})$ installed downstream of the magnet. A $\beta \sim 1$ particle traversing the $\mathrm{RICH}$ detector produces an average of about 33 hits [8]. The electromagnetic calorimeter (ECAL) [9], which provides electron pretrigger seeds and $e / \pi$ separation, is installed after the RICH and the tracking system. The ECAL is a Shashlik sampling calorimeter with $\mathrm{Pb}$ or $\mathrm{W}$ as absorber and scintillator as active material. In order to follow the steep radial dependence of the particle density, the calorimeter has been structured in three sections (Inner, Middle and Outer) with differing granularities. The Muon detector (MUON) [10] provides the muon pretrigger seeds and the muon identification, and is located in the most downstream part of the detector. It consists of four superlayers embedded in an iron loaded concrete absorber. The sensitive area close to the beam pipe is covered by pixel chambers, while in the rest of the acceptance, tube chambers are used.

The flexibility of the trigger system [11] allows the implementation of a large variety of trigger configurations. The methods described in this paper have been used to determine the integrated luminosity of the interaction trigger (IA) data sample. The IA trigger selects events with at least one inelastic interaction in the target, by requiring either that the RICH has more than a minimum number of hits (20) or that the ECAL has more than a minimum $(1 \mathrm{GeV})$ energy deposition. The total collected statistics is about 220 million events, with an average data acquisition (DAQ) rate larger than $1000 \mathrm{~Hz}$. During the data acquisition a sample of randomly trigger events (Zero-Bias) was acquired in parallel to the IA trigger at a rate of few $\mathrm{Hz}$, allowing the possibility to check the trigger acceptance and stability. Moreover the Zero-Bias event sample has been extensively used in the luminosity determination. Due to the fact that the same data stream was used both for the various physics analyses and for the determination of the recorded luminosity, the dead time of the DAQ system cancels exactly and can thus be ignored.

\section{The cross-sections}

The total $p A$ cross-section $\sigma_{\text {tot }}$ can be divided into elastic $\left(\sigma_{\mathrm{el}}\right)$ and inelastic $\left(\sigma_{\text {inel }}\right)$ contributions:

$\sigma_{\mathrm{tot}}=\sigma_{\mathrm{el}}+\sigma_{\mathrm{inel}}=\sigma_{\mathrm{el}}+\sigma_{\mathrm{mb}}+\sigma_{\mathrm{tsd}}+\sigma_{\mathrm{bsd}}+\sigma_{\mathrm{dd}}$.

In this context, the cross-section $\sigma_{\mathrm{el}}$ is regarded as the sum of the elastic $(p A \rightarrow p A)$ and quasielastic contribution $\left(p A \rightarrow p A^{*}\right)$. The inelastic cross-section includes a minimum bias part $(\mathrm{mb})$ and a diffractive part which can be further subdivided into target single diffractive (tsd, $p A \rightarrow p Y$ ), beam single diffractive (bsd, $p A \rightarrow X A$ ) and double diffractive (dd, $p A \rightarrow X Y$ ) contributions.
Table 1

Summary of the proton nucleus cross-sections for $\mathrm{C}$, Ti and $\mathrm{W}$ nuclei. Details are given in the text

\begin{tabular}{lrrr}
\hline Cross-section $(\mathrm{mb})$ & \multicolumn{1}{l}{$\mathrm{C}$} & \multicolumn{1}{l}{$\mathrm{Ti}$} & $\mathrm{W}$ \\
\hline$\sigma_{\text {tot }}$ & $351.6 \pm 4.0$ & $1045 . \pm 30$. & $2913 . \pm 43$. \\
$\sigma_{\text {inel }}$ & $250.7 \pm 2.6$ & $682.5 \pm 5.6$ & $1788 . \pm 23$. \\
$\sigma_{\text {el }}$ & $100.9 \pm 4.8$ & $362.5 \pm 30.5$ & $1125 . \pm 49$. \\
$\sigma_{\text {bsd }}$ & $8.4 \pm 1.7$ & $21.7 \pm 4.3$ & $41.2 \pm 8.2$ \\
$\sigma_{\text {tsd }}$ & $9.2 \pm 2.3$ & $14.9 \pm 3.8$ & $23.9 \pm 6.3$ \\
$\sigma_{\text {dd }}$ & $0.7 \pm 0.3$ & $1.1 \pm 0.5$ & $1.5 \pm 0.6$ \\
$\sigma_{\mathrm{mb}}$ & $232.4 \pm 3.9$ & $644.8 \pm 8.1$ & $1721 . \pm 26$. \\
\hline
\end{tabular}

The values for the total and inelastic cross-sections reported in Table 1 were obtained using the method of Ref. [12] with one exception: in order to minimize a possible systematic bias, we do not apply the $A^{\alpha}$ scaling law adopted there. Instead we use the experimental results on carbon and tungsten nuclei for the total cross-section of Ref. [13] and for the inelastic crosssections of Refs. [14-16]. All of these measurements were obtained at beam momenta ranging from 180 to $400 \mathrm{GeV} / c$ and have to be scaled to $920 \mathrm{GeV} / c$ with the prescription given in Ref. [17]. Due to the absence of data on titanium, the $A^{\alpha}$ scaling law is applied only to interpolate the $\mathrm{Al}$ and $\mathrm{Fe}$ data of the experiments quoted. The elastic cross-sections are obtained using Eq. (3). The single diffractive cross-sections are taken from Ref. [12]. The experimental results can be compared to a theoretical calculation performed in the framework of the Glauber-Gribov theory [18]. Both total and inelastic crosssections agree well within $5 \%$, while the diffractive contributions exhibit larger discrepancies. As suggested by Kopeliovich [19], an average of Model III and Model IV of Ref. [18] is used for this comparison. ${ }^{21}$ The double diffractive part, being neglected in Ref. [12], is taken from Ref. [18]. The quoted errors cover the difference between both models. The minimum bias cross-section is derived by subtracting all diffractive contributions from the inelastic cross-section with an error given by the quadratic sum of the component errors.

The total trigger efficiency can be expressed as

$\varepsilon_{\mathrm{tot}}=\frac{\varepsilon_{\mathrm{el}} \cdot \sigma_{\mathrm{el}}+\varepsilon_{\mathrm{mb}} \cdot \sigma_{\mathrm{mb}}+\varepsilon_{\mathrm{bsd}} \cdot \sigma_{\mathrm{bsd}}+\varepsilon_{\mathrm{tsd}} \cdot \sigma_{\mathrm{tsd}}+\varepsilon_{\mathrm{dd}} \cdot \sigma_{\mathrm{dd}}}{\sigma_{\mathrm{tot}}}$

where $\varepsilon_{x}$ is the efficiency for triggering on process $x$. The trigger efficiencies are determined from Monte Carlo simulation. FRITIOF $7.02[20]$ is used to generate minimum bias events in $\mathrm{pA}$ interactions, while diffractive events are generated by PYTHIA 5.7 [21] which, however, has the disadvantage of not taking into account nuclear effects. Nonetheless, since diffractive cross-sections are small compared to the minimum bias cross-section, they contribute little to the recorded sample and nuclear effects for diffractive events can be safely neglected.

\footnotetext{
${ }^{21}$ Both models are based on the saturated form of the dipole crosssection and provide a more realistic description compared to Models I and II which assume a quadratic dependence.
} 
Table 2

IA trigger efficiencies for the various processes. $K_{A}$ is defined in Eq. (5)

\begin{tabular}{llllll}
\hline Process & Generator & pp & C & Ti & W \\
\hline$\varepsilon_{\mathrm{el}}$ & PYTHIA & 0.003 & & & \\
$\varepsilon_{\text {bsd }}$ & PYTHIA & 0.583 & & & \\
$\varepsilon_{\mathrm{tsd}}$ & PYTHIA & 0.370 & & & \\
$\varepsilon_{\mathrm{dd}}$ & PYTHIA & 0.578 & & & \\
$\varepsilon_{\mathrm{mb}}$ & PYTHIA & 0.941 & & & \\
$\varepsilon_{\mathrm{mb}}$ & FRITIOF & & 0.933 & 0.953 & 0.970 \\
$\varepsilon_{\mathrm{tot}}$ & Eq. (4) & & 0.642 & 0.607 & 0.586 \\
$K_{A}$ & Eq. (5) & & 0.960 & 0.969 & 0.978 \\
\hline
\end{tabular}

The detector response is simulated by the GEANT 3.21 package [22]. Realistic detector efficiencies, readout noise and dead channels are taken into account. The simulated events are processed by the same reconstruction codes as the data. The resulting interaction trigger efficiencies are summarized in Table 2 . It has been checked that the results for the pp processes do not depend on the wire position. The small increase of the minimum bias efficiency with increasing atomic mass number is correlated to the increasing track multiplicity.

From these numbers we can conclude that the elastic contribution is negligible and that the diffractive processes are suppressed. The dominance of the minimum bias part can be illustrated by calculating its detectable fraction $K_{A}$

$K_{A}=\frac{\sigma_{\mathrm{mb}} \cdot \varepsilon_{\mathrm{mb}}}{\sigma_{\mathrm{tot}} \cdot \varepsilon_{\mathrm{tot}}}$

given in Table 2. The impact of the uncertainties on luminosities and trigger efficiencies will be discussed in Section 7.2.

\section{General remarks on the luminosity determination}

In the following, the luminosity given by Eq. (2) will be expressed in terms of the total number of events satisfying the IA trigger $\left(N_{\mathrm{IA}}\right)$, the average number of interactions per bunch crossing $\left(\lambda_{\text {tot }}\right)$, the trigger efficiency per single interaction $\left(\varepsilon_{\mathrm{tot}}\right)$ and the total hadronic cross-section $\left(\sigma_{\mathrm{tot}}\right)$. In order to do this, two assumptions are made:

- the number of interactions per filled bunch can be described by a single Poisson distribution $P\left(n, \lambda_{\text {tot }}\right)$, for all bunch crossings in a given data run:

$P\left(n, \lambda_{\mathrm{tot}}\right)=\frac{\lambda_{\mathrm{tot}}^{n} \mathrm{e}^{-\lambda_{\mathrm{tot}}}}{n !}$

- the trigger efficiency for $n$ interactions, $\left(\varepsilon_{\text {tot }}\right)_{n}$, is given by

$\left(\varepsilon_{\mathrm{tot}}\right)_{n}=1-\left(1-\varepsilon_{\mathrm{tot}}\right)^{n}$

where $\varepsilon_{\text {tot }}$ is the trigger efficiency for single interaction.

A test of the validity of the first assumption is discussed in Section 7.4, while the second assumption has been checked in Monte Carlo studies and by checking the dependence of the measured $\lambda_{\text {tot }}$ with interaction rate as measured by the target steering scintillator hodoscopes (see Section 7.5).

With these assumptions, the total number of recorded triggers resulting from interactions in the target, $N_{\mathrm{IA}}$ is given by

$$
N_{\mathrm{IA}}=N_{B X} \cdot \sum_{n=0}^{\infty}\left(P\left(n, \lambda_{\mathrm{tot}}\right) \cdot\left(\varepsilon_{\mathrm{tot}}\right)_{n}\right)=N_{B X} \cdot\left(1-\mathrm{e}^{-\varepsilon_{\mathrm{tot}} \cdot \lambda_{\mathrm{tot}}}\right)
$$

where $N_{B X}$ is the total number of $B X \mathrm{~s}$ considered. From this equation, given the general relationship of Eq. (2), we finally obtain

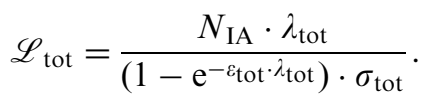

Because the product $\varepsilon \cdot \lambda$ is typically $\approx 10 \%$ for our data taking conditions, the measured luminosity is, to first order, inversely proportional to the trigger efficiency and the cross-section, while the average number of interactions, $\lambda_{\text {tot }}$, enters only as a second order correction.

In Eq. (9), $N_{\text {IA }}$ can be expressed as a function of the number of recorded triggers $\left(N_{\text {tape }}\right)$ and of the number of background events $\left(N_{\mathrm{bkg}}\right)$ :

$N_{\text {IA }}=N_{\text {tape }}-N_{\text {bkg }}=N_{\text {tape }} \cdot\left(1-f_{\text {bkg }}\right)$

where $f_{\mathrm{bkg}}$ is the fraction of background events in the sample (see Section 7.6).

Since, as discussed in Section 4, the recorded event sample is dominated by minimum bias interactions, the luminosity can be expressed as a function of minimum bias quantities

$\mathscr{L}_{\text {tot }}=\frac{N_{\text {tape }} \cdot\left(1-f_{\text {bkg }}\right) \cdot \lambda_{\mathrm{mb}}}{\left(1-\mathrm{e}^{\left.-\varepsilon_{\mathrm{mb}}{ }_{\mathrm{mb}}\right)} \cdot \sigma_{\mathrm{mb}}\right.} \cdot K_{A}$

where $K_{A}$ is defined in Section 4 and $\lambda_{\mathrm{mb}}$ is defined in Section 6 . This is the final expression which will be used to determine the luminosity for each run.

\section{The determination of $\lambda_{\mathrm{mb}}$}

The determination of $\lambda_{\mathrm{mb}}$ relies on the pseudo random trigger data sample acquired in parallel to the IA trigger and the large-acceptance detectors which constitute the spectrometer. Specifically, $\lambda_{\mathrm{mb}}$ is obtained by combining the information from a variety of subdetectors to also provide a cross-check of the stability of the result and the systematic uncertainties due to the detector response and the event model of the Monte Carlo. Only the filled bunches of HERA were considered.

The average number of IA per $B X$ can be evaluated with respect to any subdetector observable $X$ which depends linearly on the interaction rate, by exploiting the following definition:

$\lambda_{\mathrm{mb}}=-\frac{1}{\varepsilon_{\mathrm{mb}}(X)} \cdot \ln \left(1-\frac{N_{X}}{N_{B X}}\right)$ 
where $N_{X}$ is the number of events with observable $X$ above a certain threshold and $\varepsilon(X)$ is the corresponding efficiency (i.e. probability that an interaction will result in $X$ being above threshold) as evaluated from the FRITIOF simulation. To avoid possible confusion, we note that $\lambda_{\mathrm{mb}}$ as defined by Eq. (12) is close to but not equal to the average number of minimum bias interactions per $B X$. With this definition, Eq. (11) is nonetheless exact. The sample of random trigger events for all runs is sufficiently large that the statistical error is always negligible compared to the systematic error estimate. The list of the observables $X$ used for the determination of $\lambda_{\mathrm{mb}}$ is given in Table 3 . As can be seen, two subdetectors are directly involved in this method, namely RICH and ECAL, while the VDS and the OTR are indirectly involved when the number of reconstructed tracks is considered.

It is important to note that for the determination of $\lambda_{\mathrm{mb}}$, no reconstructed quantity associated only with the VDS is used. The reason for this is that all such quantities were found to be sensitive to the presence of $\delta$-rays generated by

Table 3

Description of the eight $X$ quantities used to determine $\lambda_{\mathrm{mb}}$

\begin{tabular}{ll}
\hline $\mathrm{X}$ & Description \\
\hline hrich & $\begin{array}{l}\text { Number of reconstructed hits in the } \\
\text { RICH detector } \\
\text { Total energy deposition in the ECAL } \\
\text { inner section }(\mathrm{GeV}) \\
\text { Total energy deposition in the ECAL } \\
\text { middle section }(\mathrm{GeV}) \\
\text { Total energy deposition in the ECAL } \\
\text { outer section }(\mathrm{GeV})\end{array}$ \\
$\mathrm{e}(\mathrm{ECAL}$, outer $)$ & $\begin{array}{l}\text { Total energy deposition in full ECAL } \\
\text { (GeV) }\end{array}$ \\
e(ECAL, ECAL) & $\begin{array}{l}\text { Number of reconstructed } \\
\text { electromagnetic clusters in ECAL } \\
\text { Number of hit towers in ECAL } \\
\text { nclus(ECAL) }\end{array}$ \\
humber of reconstructed tracks \\
hecal
\end{tabular}

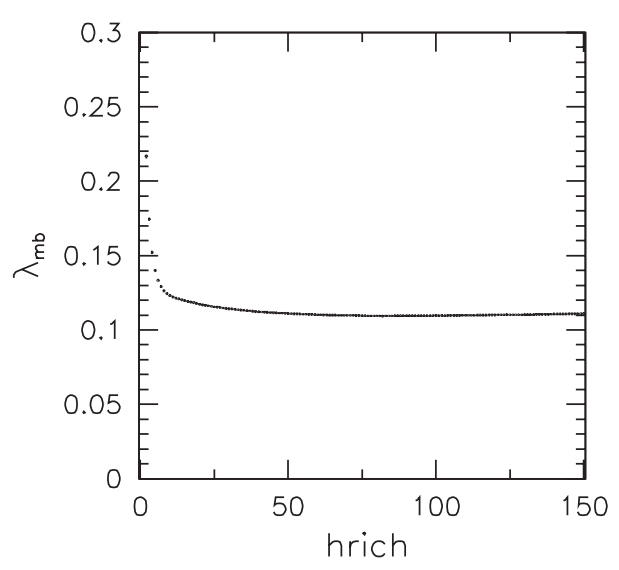

the proton beam in the target, as will be further discussed in Section 7.7.

The $\lambda$ values calculated with Eq. (12) as a function of two of the $X$ observables (namely hrich and $e(\mathrm{ECAL}$, inner)) defined in Table 3 are shown in Fig. 3. These plots show a common feature: the existence of a broad stationary point for $\lambda_{\mathrm{mb}}$. The left plot in Fig. 3 shows the $\lambda_{\mathrm{mb}}$ values obtained as a function of the threshold applied on the number of hits (hrich) seen in the RICH detector. The rise at small values of RICH hits is due to noise in the detector, while the smooth increase for large number of RICH hits is mainly due to the fact that the Monte Carlo does not precisely reproduce the RICH hit multiplicity per event, although the resulting value of $\lambda_{\mathrm{mb}}$ is nearly independent of the threshold over a wide range. This trend is confirmed, in a more or less pronounced way (see e.g. the right plot of the same Figure), also for the other variables listed in Table 3.

As a consequence, for each observable, as the best estimate of $\lambda_{\mathrm{mb}}$ the value $\left(\lambda_{\min }(X)\right)$ is taken to be its minimum value. The best evaluation of $\lambda_{\mathrm{mb}}$ is then defined as

$\lambda_{\mathrm{mb}}=\sum_{X=1,8} \frac{\lambda_{\min }(X)}{8}$.

The values of $\lambda_{\min }(X)$ obtained from all are in good agreement and their spread is used as a measure of the systematic uncertainty.

\section{Systematic uncertainties and checks}

\subsection{General considerations}

According to Eq. (11) and the assumption on which Eq. (6) is based, the following systematic uncertainties must be taken into account:

- the uncertainty on $K_{A}$, arising from the Monte Carlo (MC) event model and the poorly known observation probability of diffractive processes;

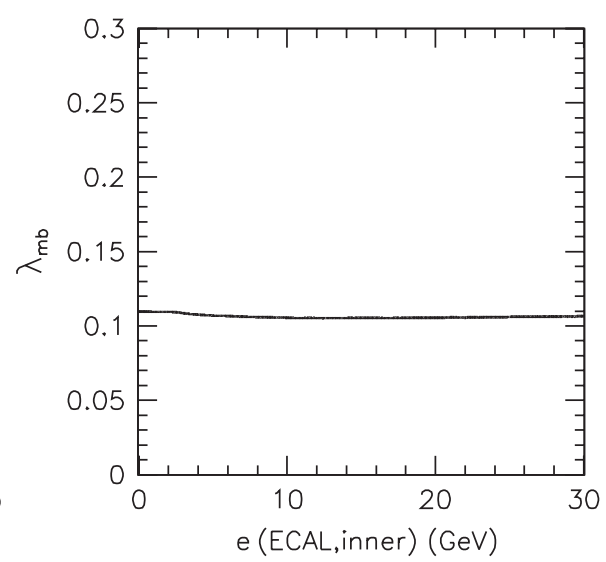

Fig. 3. Two typical distributions for the measured average number of interactions per bunch crossing as a function of the cut on different reconstructed quantities for the tungsten target wire. The distributions for RICH (number of reconstructed hits per event, -hrich-), ECAL (total energy released per event in the inner section of the calorimeter, $-e(E C A L$, inner $)-)$ are shown. 
- the uncertainty on the determination of $\lambda_{\mathrm{mb}}$;

- the uncertainty associated with deviations of the interaction probability distribution from the assumed Poisson distribution (e.g. due to the uneven filling of bunches);

- the uncertainty associated with possible out-of-time interactions (i.e. coasting beam interactions) and fake triggers from detector noise.

No systematic uncertainty due to reconstruction efficiency appears on this list since possible systematic biases due to reconstruction are included in the systematic uncertainty assigned to $\lambda_{\mathrm{mb}}$.

The determination of $\mathscr{L}_{\text {tot }}$ could, however, be biased if the on-line trigger does not operate according to expectations. Possible triggering errors are checked for by using special bits written into the event record to indicate the trigger decision. The online trigger requirements are imposed offline on the sample of random trigger events taken with each IA trigger run and compared to the the online decision. It is found that there is no significant inefficiency from the online trigger while the percentage of spurious triggers due to electronic misbehavior is typically at level of a few per thousand. Some additional masking of noisy channels is found to be necessary, although the effect on the trigger acceptance is negligible. We conclude that the trigger performed according to expectations and introduces no additional biases on the measurement of $\mathscr{L}_{\text {tot }}$.

The general expression for the squared relative uncertainty on the luminosity follows from Eq. (11):

$$
\left(\frac{\delta \mathscr{L}_{\mathrm{tot}}}{\mathscr{L}_{\mathrm{tot}}}\right)^{2}=\frac{\delta K_{A}}{K_{A}} \oplus \frac{\delta \lambda_{\mathrm{mb}}}{\lambda_{\mathrm{mb}}} \oplus\left(\frac{\delta \mathscr{L}}{\mathscr{L}}\right)_{\mathrm{bkg}} \oplus\left(\frac{\delta \mathscr{L}}{\mathscr{L}}\right)_{\text {Pois }}
$$

which is the quadratic sum of the relative systematic uncertainties on $K_{A}$ (see Section 7.2), on $\lambda_{\mathrm{mb}}$ (see Section 7.3), on the background and on the Poisson assumption (see Eq. (6)). The last two sources of systematic uncertainty will be discussed in Sections 7.4 and 7.6, respectively. Finally, to separate the measurement uncertainties from the uncertainties on the present knowledge of the total cross-section $\left(K_{A}\right.$ term), the following quantity (which will be used in Section 8) is defined:

$$
\left(\frac{\delta \mathscr{L}_{\mathrm{tot}}}{\mathscr{L}_{\mathrm{tot}}}\right)_{\mathrm{det}}^{2}=\frac{\delta \lambda_{\mathrm{mb}}}{\lambda_{\mathrm{mb}}} \oplus\left(\frac{\delta \mathscr{L}}{\mathscr{L}}\right)_{\mathrm{bkg}} \oplus\left(\frac{\delta \mathscr{L}}{\mathscr{L}}\right)_{\mathrm{Pois}} .
$$

\subsection{Uncertainty on $K_{A}$}

The relative uncertainty $\left(\left(\delta K_{A}\right) / K_{A}\right)$ on the detectable fraction $K_{A}$ (defined in Eq. (5)) depends on the uncertainties of the cross-sections, quoted in Table 1 , and on the trigger efficiencies of the various production processes. Given the poor knowledge of the structure of final states produced by diffractive processes, the trigger efficiencies are assumed to be fully unknown but limited to the range
Table 4

Detectable fractions and their relative uncertainty for carbon, titanium and tungsten

\begin{tabular}{lll}
\hline & $K_{A}$ & $\left(\delta K_{A}\right) / K_{A}$ \\
\hline $\mathrm{C}$ & 0.960 & 0.023 \\
$\mathrm{Ti}$ & 0.969 & 0.018 \\
$\mathrm{~W}$ & 0.978 & 0.016 \\
\hline
\end{tabular}

from 0 to 1 . Thus, an error of $\sigma_{\varepsilon_{\mathrm{bsd}}}=\sigma_{\varepsilon_{\mathrm{tsd}}}=\sigma_{\varepsilon_{\mathrm{dd}}}=\frac{1}{\sqrt{12}}$ is assigned. The error on $\varepsilon_{\mathrm{mb}}$ is not included here as discussed in Section 7.1. The resulting uncertainties are summarized in Table 4.

\subsection{Uncertainty on $\lambda_{\mathrm{mb}}$}

The method used to determine $\lambda_{\mathrm{mb}}$ is influenced by the Monte Carlo description of the HERA-B detector as well as the event model of the event generator. The resulting uncertainty on $\lambda_{\mathrm{mb}}$ is taken to be the rms spread of the $\lambda_{\mathrm{mb}}$ values calculated with Eq. (13). The typical values obtained are

$\frac{\delta_{\lambda_{\mathrm{mb}}}}{\lambda_{\mathrm{mb}}} \simeq 0.04$

or better, depending on the target material.

Possible sources of systematic uncertainty related to the way $\lambda_{\mathrm{mb}}$ is determined have been investigated. For example, examination of the observables given in Table 3 shows that six of them involve ECAL and are thus possibly subject to correlated systematic effects while RICH and VDS + OTR appear only with one variable each. For this reason an alternative quantity $\lambda_{\mathrm{mb}}^{\prime}$ is defined as

$\lambda_{\mathrm{mb}}^{\prime}=\frac{\lambda_{\mathrm{opt}}(1)+\sum_{X=2,7} \frac{\lambda_{\mathrm{opt}}(X)}{6}+\lambda_{\mathrm{opt}}(8)}{3}$.

The relative discrepancy of $\lambda_{\mathrm{mb}}$ and $\lambda_{\mathrm{mb}}^{\prime}$ is given by

$\left(\frac{\delta \lambda_{\mathrm{mb}}}{\lambda_{\mathrm{mb}}}\right)_{\text {method }}=\frac{\lambda_{\mathrm{mb}}-\lambda_{\mathrm{mb}}^{\prime}}{\lambda_{\mathrm{mb}}}$.

The mean value of the distribution of this quantity is found to be statistically compatible with zero and its rms width is $\approx 0.006$ or better, depending on target material. The smallness of this term, compared to the overall systematic uncertainty on $\lambda_{\mathrm{mb}}$ (see Eq. (16)), shows that the calculation of $\lambda_{\mathrm{mb}}$ is insensitive to the relative weights given to the various methods.

\subsection{Uncertainty on the distribution of the number of IA per $B X$}

One important assumption is that the number of interactions per HERA machine bunch follows a Poisson distribution (see Eq. (6)). In order to evaluate the systematic uncertainty associated to the non-Poisson behavior of the $B X$ population, the total luminosity can 
alternatively be measured for each run, also as a sum over all the $B X$ contributions, i.e.:

$\left(\mathscr{L}_{\mathrm{tot}}\right)_{B X}=\frac{K_{A}}{\sigma_{\mathrm{mb}}} \cdot \sum_{i=1}^{180} \frac{N_{\mathrm{IA}_{i}} \cdot \lambda_{m b_{i}}}{\left(1-\mathrm{e}^{-\varepsilon_{\mathrm{mb}} \lambda_{m b}}\right)}$.

The quantity $\left(\mathscr{L}_{\text {tot }}\right)_{B X}$ is then compared with the total luminosity calculated according to the basic procedure (see Eq. (11)). In this way, we can define the systematic uncertainty due to the non Poisson behavior of the beam as

$\left(\frac{\delta \mathscr{L}}{\mathscr{L}}\right)_{\text {Pois }}=\frac{\mathscr{L}_{\text {tot }}-\left(\mathscr{L}_{\text {tot }}\right)_{B X}}{\mathscr{L}_{\text {tot }}}$

The mean of the distribution of this quantity for all the runs with more than $3 \times 10^{5}$ events shows a slight shift $(\approx 0.4 \%)$ toward negative values. The rms of the distribution is $\approx 0.009$ or better, depending on target material.

\subsection{The dependence of $\lambda_{\mathrm{mb}}$ on the target interaction rate}

The dependence of $\lambda_{\mathrm{mb}}$, as determined by the method of Section 6 , on the target steering hodoscope rate $\left(R_{\text {hod }}\right)$ was checked. In general the dependence should be linear at sufficiently low interaction rate.

For each of the three target materials $(\mathrm{C}, \mathrm{Ti}$ and $\mathrm{W}), \lambda_{\mathrm{mb}}$ was determined for hodoscope interaction rates of $0.3,0.5,1,3,5$ and $10 \mathrm{MHz}$. For each target, the resulting $\lambda_{\mathrm{mb}}$ values, excluding the $10 \mathrm{MHz}$ point, were fit to a straight line. The results of the fit for carbon and tungsten target wires are shown in Fig. 4. The $10 \mathrm{MHz}$ point lies below the fit line indicating possible saturation of the hodoscopes or possibly a breakdown of the Poisson assumption at high interaction rates. The normalized $\chi^{2}$ of the linear fit is about one or better (for all three sets of runs) and indicates a linear relation between interaction rates measured by two very different techniques for rates up to $5 \mathrm{MHz}$. This in turn supports the two assumptions made in Section 5 since the hodoscope rates do not rely on the Poisson assumption and compensating non-linearities in the two methods are unlikely.

\subsection{Background estimate}

As shown in Eq. (10), the number of recorded events must be corrected for background events either from fake triggers (e.g. electronic noise in the $\mathrm{RICH}$ ) or from coasting beam interactions (beam-gas interactions and background from interactions upstream of the target are negligible). The best method to determine the fraction of background events is to compare empty and filled bunches using the random trigger events. For this purpose the interaction trigger requirements are applied offline to the random trigger events which are equally distributed over all 220 bunches. Normalizing to the 180 filled bunches, we obtain

$f_{\text {bkg }}^{\prime}=\frac{180}{40} \times\left(\frac{N_{\text {tape }}(\text { empty } B X)}{N_{\text {tape }}(\text { filled } B X)}\right)$.

A small (approximately 5\%) correction is made to account for "in-time" coasting beam interactions which are well synchronized in time with the detector's integration gates and thus are no different from ordinary interactions from bunched beam protons.

The resulting values for $f_{\mathrm{bkg}}$ from the random trigger sample are summarized in Table 5 and used in Eq. (11). To estimate the uncertainty on $f_{\mathrm{bkg}}$, the software trigger thresholds are varied over a wide range. The uncertainty,

Table 5

The fraction of background events $f_{\text {bkg }}$ for each target wire and the relative systematic uncertainty

\begin{tabular}{lcc}
\hline & $f_{\text {bkg }}$ & $\left(\frac{\delta \mathscr{L}}{\mathscr{L}}\right)_{\mathrm{bkg}}$ \\
\hline $\mathrm{C}$ & 0.031 & 0.018 \\
$\mathrm{Ti}$ & 0.057 & 0.023 \\
$\mathrm{~W}$ & 0.026 & 0.019 \\
\hline
\end{tabular}
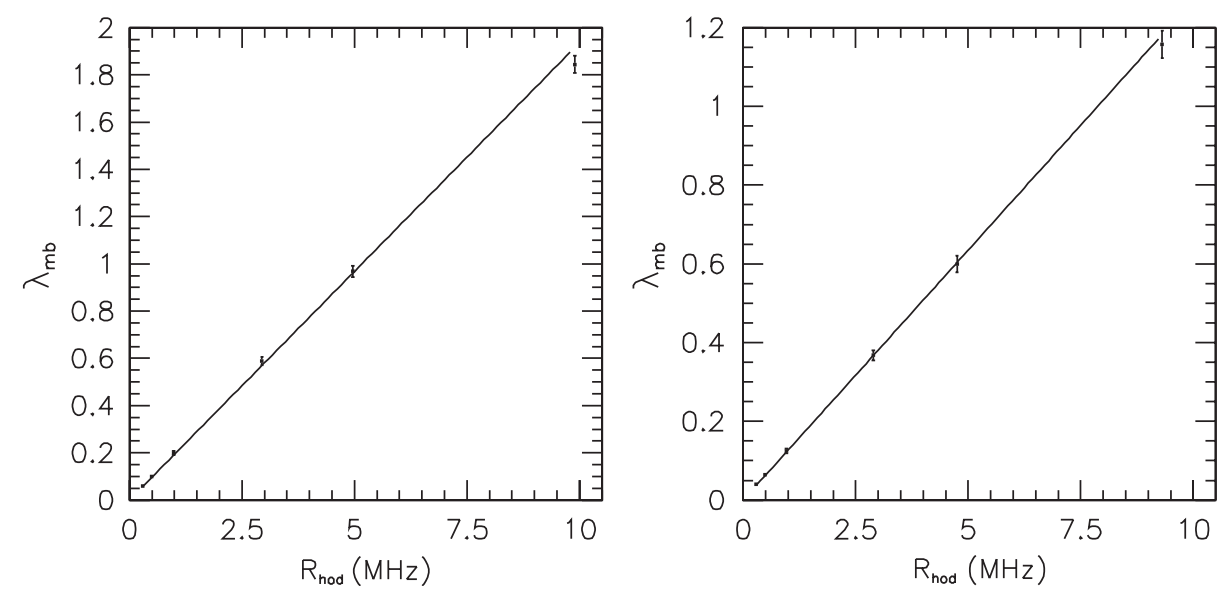

Fig. 4. Test of linear dependence of the $\lambda_{\mathrm{mb}}$ values determined with the method described in Section 6 with respect to the target interaction rate measured by the scintillator hodoscope system for the carbon (left plot) and tungsten (right plot) target wires. 
$\delta \mathscr{L}_{\mathrm{bkg}}$, is obtained by dividing the difference of the extreme values by $\sqrt{12}$. The uncertainties due to the in-time coasting beam correction discussed above are negligible compared to the uncertainties given in the table.

\subsection{Production of $\delta$-rays in the target}

The presence of $\delta$-rays in the data sample (see Section 6) is both a nuisance, since it compromises the VDS based methods, and an opportunity for a systematic check of the luminosity calculation, since the luminosity can be estimated from the observed rate of $\delta$-ray production. The results of a study of $\delta$-ray production applied to a run taken with the carbon target sample are presented and compared to the luminosity estimates given in Section 6 . With further development, the techniques presented here could be used for a precise luminosity determination in experiments using thin targets.

The luminosity for a fixed-target experiment in a proton beam is proportional to the sum of target path lengths of all protons $\left(N_{\text {tot }}\right)$ which traverse the target:

$\mathscr{L}=\frac{\rho N_{\mathrm{A}}}{A} \cdot \sum_{i=1}^{N_{\mathrm{tot}}} z_{i}$

where $A$ is the atomic mass of the target material, $N_{\mathrm{A}}$ is Avogadro's number, $\rho$ is the target density in $\left(\mathrm{g} / \mathrm{cm}^{3}\right)$ and $z_{i}$ is the length of the target traversed by the $i$ th proton.

The number of $\delta$-rays $\left(N_{\delta \text {,prod }}\right)$ produced in a kinetic energy ( $T$ ) interval from $T_{\min }$ to $T_{\max }$ is proportional to the same summed target length [17]:

$$
\begin{aligned}
N_{\delta, \text { prod }} & =0.154 \frac{Z}{A} \rho \sum_{i=1}^{N_{\text {tot }}} z_{i} \cdot \int_{T_{\min }}^{T_{\max }} \frac{\mathrm{d} T}{T^{2}} \\
& \approx 0.154 \frac{Z}{A} \frac{\rho}{T_{\min }} \sum_{i=1}^{N_{\text {tot }}} z_{i}
\end{aligned}
$$

where $Z, A$, and $\rho$ are the atomic number, atomic mass and density (in $\mathrm{g} / \mathrm{cm}^{3}$ ) of the target and $T$ is in $\mathrm{MeV} . T_{\max }$ is approximately $475 \mathrm{GeV}$ for $920 \mathrm{GeV}$ incident protons.

Combining Eqs. (22) and (23) results in the following equation relating luminosity to the number of produced $\delta$-rays:

$\mathscr{L}=\frac{N_{\mathrm{A}} T_{\min }}{0.154 Z} \cdot N_{\delta, \text { prod }}=\frac{N_{\mathrm{A}} T_{\min }}{0.154 Z} \cdot \frac{N_{\delta, \mathrm{obs}}}{\varepsilon_{\delta}}$

where $N_{\delta \text {,obs }}$ is the number of observed $\delta$-rays and $\varepsilon_{\delta}$ is the average probability that the $\delta$-ray escapes the target and is reconstructed.

The $\delta$-ray detection efficiency $\left(\varepsilon_{\delta}\right)$ is evaluated by Monte Carlo. The $\delta$-rays are generated according to Equations 27.5 and 27.6 of Ref. [17] with a minimum kinetic energy threshold of $1 \mathrm{MeV}$ and tracked through the target and detector using the GEANT3-based [22] HERA-B simulation program. Since the VDS has acceptance for tracks from the target in the polar angular interval $0.01 \lesssim \theta \lesssim 0.7 \mathrm{rad}$, corresponding to a $\delta$-ray momentum range of $1.88 \lesssim p_{\delta} \lesssim 10220 \mathrm{MeV} / c$ (see Equation 27.6 of Ref. [17]), the $1 \mathrm{MeV}$ kinetic energy threshold corresponds to $\delta$-rays which are well outside the detector acceptance. The generated Monte Carlo events are subjected to the same reconstruction and analysis code (see below) used for the data.

An average efficiency of $\approx 7 \%$ after all cuts is found. We estimate a $15 \%$ relative systematic uncertainty on this number coming from uncertainties in the material distribution in the vertex detector and from sensitivity to Monte Carlo parameters, in particular to the minimum kinetic energy cutoff for tracking by GEANT, nominally set to $30 \mathrm{keV}$. (These sources of systematic error could in principle be greatly reduced by a more precise inventory of detector materials and by a more thorough study of the tracking of very low momentum electrons.)

Candidate $\delta$-rays are reconstructed using the standard HERA-B VDS reconstruction software applied to a pseudorandom-triggered carbon target run and then searched for in events from filled bunch crossings which do not pass the IA trigger condition. Distributions of track parameters and derived quantities from the $\delta$-ray Monte Carlo and data are found to be in close agreement when segments which extrapolate to near the average vertex position of hadronic interactions are removed. Such tracks are typically high momentum tracks from hadronic $p N$ interactions.

We define the impact parameter of a track as the difference between the average position of vertices from
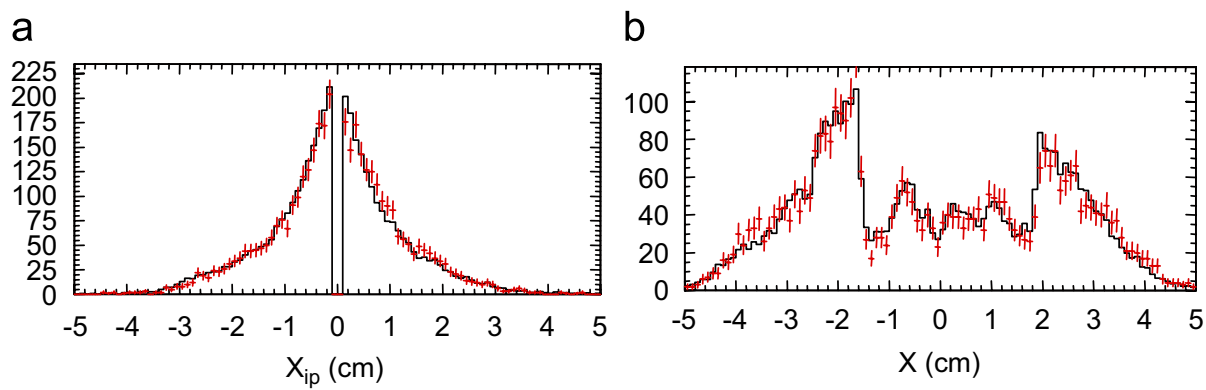

Fig. 5. (a) The distribution of $X_{\text {ip }}$ for selected tracks (see text). (b) The distribution of $X$-view impact points on the first VDS detector layer, for the same tracks used in part a. In both cases, the data is indicated by the histogram and the Monte Carlo by the points with error bars. 
hadronic interactions (and therefore the average impact point of the beam on the wire target) and the track's position when extrapolated to the $Z$-position of the target. Fig. 5(a) shows the $X$-view impact parameter $\left(X_{\text {ip }}\right)$ distribution of reconstructed VDS segments in non-IA events which contain a single reconstructed segment with $\left|X_{\text {ip }}\right|>1 \mathrm{~mm}$ which originates in the first VDS layer. The cut on $X_{\text {ip }}$ removes a signal from high-momentum tracks from hadronic $p N$ interactions (approximately $\frac{1}{4}$ of the removed tracks form a narrow peak above the relatively broad distribution shown in the figure). The data, indicated by the histogram, and the Monte Carlo, indicated by the points with error bars, are in good agreement. Note that the width of the $X_{\text {ip }}$ distribution is largely determined by multiple scattering in the VDS and therefore depends on the momentum of the reconstructed tracks. The close match between data and Monte Carlo implies that the momentum spectra of reconstructed tracks in data and the $\delta$-ray Monte Carlo are similar. Fig. 5(b), showing the distribution of the $X$-view impact points of the same tracks used in Fig. 5(a) at the first VDS layer, also illustrates the good agreement between data and Monte Carlo. The corresponding distributions in the $Y$-view also agree well with each other as do the distributions of track polar angles. The overall close agreement strongly suggests that the observed tracks are indeed caused by $\delta$-rays originating in the target. Further evidence that the observed tracks are associated with beam protons traversing the target comes from the greatly reduced rate of such tracks in empty bunch crossings: $\approx 3 \%$ of the rate in filled bunch crossings.

The observed rate of $\delta$-ray candidates is $0.068 \pm 0.003$ per $B X$. When the same event and track selection criteria are applied to the minimum bias Monte Carlo, a rate of $5 \times 10^{-4}$ candidates per interaction is observed, or approximately $10^{-4}$ candidates per $B X$ for the analyzed run. The rate of target single diffractive events $(0.004$ per $B X$, see Table 1) is also small compared to the observed rate. We conclude that the observed tracks cannot be due to hadronic interactions.

Assuming that the observed tracks are indeed $\delta$-rays, Eq. (24) gives a luminosity estimate of $633 \pm 28 \pm 95 \mathrm{mb}^{-1}$ per $B X$, to be compared with the luminosity estimate of $688 \pm 35 \mathrm{mb}^{-1}$ computed using the method described in Section 6. The agreement within errors lends further credence to the hypothesis that the tracks described in this section are $\delta$-rays from the target and also serves as a crosscheck of the method of Section 6.

\section{Summary and conclusions}

As previously noted the uncertainties affecting the total luminosity measurement are dominated by the systematic contribution, since each IA trigger run contains enough random trigger events to make the contribution from statistics negligible. In Table 6 we summarize the overall relative uncertainty on the total luminosity calculation $\left(\left(\delta \mathscr{L}_{\text {tot }}\right) / \mathscr{L}_{\text {tot }}\right)$. In the second column the uncertainty on
Table 6

Values of the contributions to the relative systematic uncertainty and overall relative systematic uncertainty on the total luminosity calculation $\left(\left(\delta \mathscr{L}_{\text {tot }}\right) \mathscr{L}_{\text {tot }}\right)$

\begin{tabular}{llllllll}
\hline & $\frac{\delta K_{A}}{K_{A}}$ & $\frac{\delta \lambda_{\mathrm{mb}}}{\lambda_{\mathrm{mb}}}$ & $\left(\frac{\delta \mathscr{L}}{\mathscr{L}}\right)_{\mathrm{Pois}}$ & $\left(\frac{\delta \mathscr{L}}{\mathscr{L}}\right)_{\mathrm{bkg}}$ & $\left(\frac{\delta \mathscr{L}_{\mathrm{tot}}}{\mathscr{L}_{\mathrm{tot}}}\right)_{\mathrm{det}}$ & $\frac{\delta \mathscr{L}_{\mathrm{tot}}}{\mathscr{L}_{\mathrm{tot}}}$ & $\left(\frac{\delta \mathscr{L}_{\mathrm{tot}}}{\mathscr{L}_{\mathrm{tot}}}\right)_{\mathrm{uc}}$ \\
\hline $\mathrm{C}$ & 0.023 & 0.039 & 0.009 & 0.018 & 0.044 & 0.050 & 0.039 \\
$\mathrm{Ti}$ & 0.018 & 0.042 & 0.009 & 0.023 & 0.049 & 0.052 & 0.042 \\
$\mathrm{~W}$ & 0.016 & 0.032 & 0.010 & 0.019 & 0.039 & 0.042 & 0.029 \\
\hline
\end{tabular}

The uncorrelated part $\left(\left(\delta \mathscr{L}_{\text {tot }}\right) / \mathscr{L}_{\text {tot }}\right)_{\mathrm{uc}}$ is given in the last column.

$K_{A}$ is given. This contribution depends on the present knowledge of the cross-sections (see Table 1) and can in principle be improved in the future. The following three columns list individual contributions to the systematic uncertainty on detection which are combined according to Eq. (15) to give the total detection uncertainties shown in the sixth column.

When the method described in this paper is applied to the 2002 HERA-B minimum bias data taking period, the following integrated luminosities are obtained for each of the three target materials:

$$
\begin{aligned}
& \mathscr{L}_{\text {tot, } \mathrm{C}}=405.8 \pm 9.3 \pm 17.9 \mu \mathrm{b}^{-1} \\
& \mathscr{L}_{\text {tot } \mathrm{Ti}}=30.9 \pm 0.6 \pm 1.5 \mu \mathrm{b}^{-1} \\
& \mathscr{L}_{\text {tot }, \mathrm{W}}=38.3 \pm 0.6 \pm 1.5 \mu \mathrm{b}^{-1}
\end{aligned}
$$

where the first error corresponds to the uncertainty on $K_{A}$ and second summarizes the remaining uncertainty mainly due to the HERA-B experimental conditions. The overall systematic uncertainty can then be obtained as the quadratic combinations of these two terms.

The method for luminosity measurement described in this paper is based on the determination of the average number of interactions per bunch crossing, $\lambda$, and on the knowledge of the total interaction cross-section $\sigma$ (see Eq. (2)). The availability of a small fraction (few percent) of events acquired in parallel to the main stream of data with a completely unbiased trigger (pseudo-random trigger) was used to evaluate $\lambda$ on run by run basis.

The measurement of $\lambda$ has been performed by exploiting the information from a variety of subdetectors, without the use of any dedicated device. This strategy allowed to perform consistency checks and to obtain a conservative determination of the systematic uncertainties of the measurement.

In case the three data sets will be combined to determine the A-dependence of a cross-section, possible correlations between the systematic errors have to be taken into account. The correlated error is dominated by the uncertainty on $\lambda_{\mathrm{mb}}$ and the background correction and is estimated to be $\sim 3 \%$. The correlation coefficients vary between 0.90 and 0.92 for pairs of wires. ${ }^{22}$ The

\footnotetext{
${ }^{22}$ At the time of publication of Refs. [23,24], the correlated error was estimated to be $2 \%$, rather than the updated and more accurate value
} 
uncorrelated part of the systematic uncertainty $\left(\left(\delta \mathscr{L}_{\text {tot }}\right) / \mathscr{L}_{\text {tot }}\right)_{\text {uc }}$ can be found in the last column of Table 6.

The same strategy could be applied in future experiments such as those under construction at the LHC at CERN in Geneva, once the corresponding cross-sections have been measured.

\section{Acknowledgments}

We are grateful to the DESY laboratory and to the DESY accelerator group for their strong support since the conception of the HERA-B experiment. The HERA-B experiment would not have been possible without the enormous effort and commitment of our technical and administrative staff.

\section{References}

[1] E. Hartouni, et al., An Experiment to Study CP Violation in the $B$ System Using an Internal Target at the HERA Proton Ring, Design Report, DESY-PRC 95/01, 1995.

[2] The HERA-B Collaboration, Report on Status and prospects, DESY-PRC 00/04 (October 2000).

[3] K. Ehret, Nucl. Instr. and Meth. A 446 (2000) 190.

[4] K. Ehret, et al., Nucl. Instr. and Meth. A 456 (2001) 206.
[5] C. Bauer, et al., Nucl. Instr. and Meth. A 501 (2003) 39.

[6] T. Zeuner, et al., Nucl. Instr. and Meth. A 446 (2000) 324; Y. Bagaturia, et al., Nucl. Instr. and Meth. A 490 (2002) 223.

[7] H. Albrecht, et al., Nucl. Instr. and Meth. A 541 (2005) 610; H. Albrecht, et al., Nucl. Instr. and Meth. A 555 (2005) 310.

[8] I. Ariño, et al., Nucl. Instr. and Meth. A 516 (2004) 445.

[9] A. Zoccoli, et al., Nucl. Instr. and Meth. A 446 (2000) 246; G. Avoni, et al., Nucl. Instr. and Meth. A 461 (2001) 332.

[10] V. Eiges, et al., Nucl. Instr. and Meth. A 461 (2001) 104.

[11] V. Balagura, et al., Nucl. Instr. and Meth. A 494 (2002) 526; T. Nunez, et al., Nucl. Phys. B Proc. (Suppl.) 120 (2003) 166; M. Dam, et al., Nucl. Instr. and Meth. A 525 (2004) 566.

[12] J. Carvalho, Nuclear Physics A 725 (2003) 269.

[13] P.V.R. Murthy, et al., Nucl. Phys. B 92 (1975) 269.

[14] A.S. Carroll, et al., Phys. Lett. B 80 (1979) 319.

[15] F. Fumuro, et al., Nucl. Phys. B 152 (1979) 376.

[16] T.J. Roberts, et al., Nucl. Phys. B 159 (1979) 56.

[17] W.-M. Yao, et al., J. Phys. G 33 (2006) 1.

[18] B.Z. Kopeliovich, I.K. Potashnikova, I. Schmidt, Phys. Rev. C 73 (2006) 034901.

[19] B.Z. Kopeliovich, Private communication.

[20] H. Pi, Comp. Phys. Comm. 71 (1992) 173.

[21] T. Sjöstrand, Comp. Phys. Comm. 82 (1994) 74.

[22] R. Brun, et al., GEANT3, Internal Report CERN-DD/EE/84-1, CERN, 1987.

[23] I. Abt, (HERA-B Collaboration), et al., Phys. Lett. B 638 (2006) 407.

[24] I. Abt, (HERA-B Collaboration), et al., Eur. Phys. J. C 50 (2007) 315. 